1 Fundação Oswaldo Cruz (Fiocruz), Instituto de Informação e Comunicação Científica e Tecnologia em Saúde (Icict), Laboratório de Informação em Saúde (LIS) - Rio de Janeiro (RJ), Brasil.

diegoricardox@gmail.com

2 Fundação Oswaldo Cruz (Fiocruz), Instituto de

Informação e Comunicação Científica e Tecnologia em Saúde (Icict), Laboratório de Informação em Saúde (LIS) - Rio de Janeiro (RJ), Brasil.

ricardo.dantas@icict.fiocruz.br

3 Fundação Oswaldo Cruz (Fiocruz), Instituto de Informação e Comunicação Científica e Tecnologia em Saúde (Icict), Laboratório de Informação em Saúde (LIS) - Rio de Janeiro (RJ), Brasil.

vanderlei.matos@gmail.com

4 Fundação Oswaldo Cruz (Fiocruz), Instituto de Informação e Comunicação Científica e Tecnologia em Saúde (Icict), Laboratório de Informação em Saúde (LIS) - Rio de Janeiro (RJ), Brasil.

fviacava@gmail.com

5 Fundação Oswaldo Cruz (Fiocruz), Instituto de Informação e Comunicação Científica e Tecnologia em Saúde (Icict), Laboratório de Informação em Saúde (LIS) - Rio de Janeiro (RJ), Brasil.

carolinaccarvalho@gmail.com

\section{Cobertura de mamografias, alocação e uso de equipamentos nas Regiões de Saúde}

\author{
Mammograms coverage, allocation and use of equipment in the \\ Health Regions
}

\author{
Diego Ricardo Xavier ${ }^{\mathbf{1}}$ Ricardo Antunes Dantas de Oliveira $\mathbf{2}$, Vanderlei Pascoal de Matos ${ }^{\mathbf{3}}$, \\ Francisco Viacava4, Carolina de Campos Carvalho $\mathbf{5}$
}

\begin{abstract}
RESUMO O rastreamento por intermédio da mamografia é o principal meio de detecção da neoplasia maligna da mama. Considera-se relevante compreender como este tem se dado nas Regiões de Saúde brasileiras, unidade espacial fundamental na atual política de saúde. A análise de indicadores relacionados à cobertura de mamografia das mulheres de 40 a 69 anos, número, distribuição e grau de utilização dos mamógrafos demonstra grandes problemas, que não podem ser simplesmente atribuídos à falta de equipamentos. Destaca-se também a diversidade de situações das Regiões de Saúde e das unidades da federação, caracterizada especialmente pelas melhores condições no Sul e Sudeste e piores nas outras regiões.
\end{abstract}

PALAVRAS-CHAVE Mamografia. Instrumentação. Parâmetros. Regionalização.

ABSTRACT Mammography screening is the most important means to detect breast cancer. It is relevant to understand how this screening has been performed in Brazilian Health Regions, which are the fundamental spatial unity in the current health policy. The analysis of indicators related to the coverage of women from 40 to 69 years of age, number, distribution and degree of usage of mammography equipment show great problems in covering the targeted population, which cannot be explained only by the lack of equipment. The diversity of situations of the Health Regions and federal units is highlighted, showing better conditions in the Southeast and South and worse in the other regions.

KEYWORDS Mammography. Instrumentation. Parameters. Regional health planning. 


\section{Introdução}

O rastreamento por intermédio de mamografias é o principal meio de detecção precoce para diagnóstico de neoplasias malignas da mama, uma das principais causas de óbitos no Brasil, representando 2,6\% dos óbitos femininos. Entre 2009 e 2013, ocorreram 65.695 óbitos causados por neoplasia maligna da mama, e somente no ano de 2012 foram 13.591 óbitos. Na população feminina de 40 a 69 anos, para a qual há parâmetros definidos pelo Ministério da Saúde quanto à necessidade de diagnóstico precoce, a neoplasia maligna da mama foi a terceira principal causa de óbitos entre 2009 e 2013, com 41.490 óbitos, $5,2 \%$, do total nesse grupo. No ano de 2012, foram 8.561 óbitos, $20 \%$ dos ocorridos no período (BRASIL, 2015B).

Atualmente, no Sistema Único de Saúde (SUS), tem-se discutido a regionalização como estrutura básica da organização dos serviços de saúde. De acordo com LimaCosta e Matos (2012), a ideia de Regiões de Saúde no SUS aparece pela primeira vez na Norma Operacional de Assistência à Saúde (Noas) de 2000, na qual a regionalização é definida como a estratégia para aprimorar a descentralização dos serviços de saúde conforme previsto na Lei $n^{\circ}$ 8.080/1990 que regulamenta o SUS. O Pacto de Saúde, de 2006, reafirma a importância da regionalização a partir de seu conteúdo político, com o foco na negociação e pactuação intergovernamental. A Portaria $\mathrm{n}^{0} 4.729 / 2010$ define as Regiões de Saúde como áreas de abrangência territorial e populacional sob responsabilidade das redes de atenção especificadas (BRASIL, 2010), enquanto o Decreto Federal $n^{0}$ 7.508/2011 estabelece instrumentos para a efetivação das redes, caracterizando as regiões como espaços geográficos contínuos definidos

a partir de identidades culturais, econômicas e sociais e de redes de comunicação e infraestrutura de transportes compartilhados, com a finalidade de integrar a organização, o planejamento e a execução de ações e serviços de saúde. (BRASIL, 2011).

A relevância das Regiões de Saúde na organização dos serviços de saúde no País levou o Projeto Avaliação do Desempenho de Sistemas de Saúde (Proadess) (VIACAVA ET AL., 2004, 2012) a incorporá-las como unidades de análise do desempenho do sistema de saúde. Nesse sentido, os indicadores que eram analisados no âmbito de Unidades da Federação (UFs) foram calculados para as Regiões de Saúde, o que permite análises mais direcionadas para o planejamento e intervenção.

A importância do rastreamento para detecção precoce da neoplasia maligna da mama é ressaltada não apenas nos parâmetros do Ministério da Saúde (BRASIL, 2015B), mas também por autores como Gebrim e Quadros (2006), Oliveira et al. (2011), Silva (2012) e Silva et al. (2014). Assim, é relevante compreender como tem-se dado o rastreamento do câncer de mama sob a perspectiva da conformação das Regiões de Saúde brasileiras, unidade espacial de extrema relevância na atual política de saúde.

A análise do grau de cobertura da mamografia possibilita uma avaliação da resposta das Regiões de Saúde às demandas, que podem ser equacionadas e solucionadas pelo compartilhamento de recursos dos municípios que as compõem, além de avaliar se as Regiões de Saúde foram definidas de modo a otimizar os recursos e o acesso aos serviços de saúde.

Considera-se que há uma diversidade de situações quanto à disponibilidade/utilização dos equipamentos de mamografia, que envolvem várias dificuldades na organização do serviço, tais como a falta de equipamentos disponíveis, profissionais habilitados, subutilização de equipamentos e dificuldade de acesso em função das distâncias geográficas. Os problemas de cobertura de mamografias na população-alvo são resultantes da combinação dessas diferentes situações.

Nesse contexto, este estudo busca avaliar 
como tem ocorrido o rastreamento do câncer de mama nas Regiões de Saúde brasileiras por meio da realização periódica de mamografia na população feminina de 40 a 69 anos, grupo mais vulnerável à neoplasia maligna de mama.

\section{Métodos}

A avaliação da cobertura de mamografias foi feita considerando as necessidades populacionais e distribuição espacial, grau de utilização e adequação da oferta dos mamógrafos nas Regiões de Saúde, segundo parâmetro mais recente sugerido pelo Ministério da Saúde (BRASIL, 2009, 2015B). Com base nos princípios de universalidade, cobertura integral e acesso igualitário às ações e serviços de saúde do SUS, este estudo busca evidenciar a aplicação prática desses princípios, considerando como cobertura a realização de exames de mamografias na população-alvo definida segundo o parâmetro.

Como fonte de dados, foram utilizadas informações sobre: procedimentos 'mamografia bilateral' e 'mamografia bilateral para rastreamento' (códigos: 0204030030, 0204030188), obtidas por meio do Sistema de Informações Ambulatoriais do SUS (SIA); número de mamógrafos, registrados no Cadastro Nacional de Estabelecimentos de Saúde (CNES) e dados populacionais do Instituto Brasileiro de Geografia e Estatísticas (IBGE). O ano considerado no estudo foi 2012, por ser a última projeção populacional do IBGE para municípios, por sexo e faixas etárias, permitindo a construção do denominador dos indicadores para as Regiões de Saúde.

Como critério para elegibilidade da população, consideraram-se as mulheres de 40 a 69 anos que não dispõem de planos de saúde com as segmentações 'ambulatorial', 'hospitalar sem e com obstetrícia' ou 'plano de referência', a partir dos dados da Agência Nacional de Saúde Suplementar
(ANS, 2013). Segundo o Instituto Nacional do Câncer (Inca), mulheres entre 50 e 69 anos devem realizar a mamografia bilateral a cada dois anos (BRASIL, 2009). Entretanto, a Lei $11.664 / 2008$ determina que o SUS deve assegurar a realização de exame mamográfico a todas as mulheres a partir dos 40 anos de idade. Assim, as duas faixas etárias são consideradas pelo parâmetro mais atual para cálculo de equipamentos de mamografia do Ministério da Saúde (BRASIL, 2015B), que define que o número de mamógrafos deve estar atrelado à necessidade de exames na população residente. A partir dessa recomendação, foi elaborada a fórmula abaixo:

$$
\mathrm{Nm}=\operatorname{Pe} 1(\mathrm{D} 1+\mathrm{Ou})+\mathrm{Pe} 2(\mathrm{R} 1+\mathrm{D} 2)
$$

Em que:

$\mathrm{Nm}=$ Necessidade de mamografia (número anual estimado de mamografias necessárias).

Pel = Diferença entre a população total de mulheres com idade de 40 a 49 anos e a população nessa faixa etária coberta com plano de saúde.

Pe2 = Diferença entre a população total de mulheres com idade de 50 a 69 anos e a população nessa faixa etária coberta com plano de saúde.

D1 $=10 \%$ da população de mulheres de 40 a 49 anos (indicação de mamografia diagnóstica).

$\mathrm{Ou}=10 \%$ da população de mulheres de 40 a 49 anos para outras indicações.

$\mathrm{R} 1=50 \%$ da população de mulheres de 50 a 69 anos (indicação de rastreamento).

D2 $=8,9 \%$ da população feminina de 50 a 69 (indicação diagnóstica). (ADAPTADO DE BRASIL, 2009, 2015).

Para estimar o número de mamógrafos necessários, com base nas necessidades anuais de mamografias da população, admitiu-se que cada equipamento deve realizar anualmente 5.069 exames, considerando 3 exames/hora $\mathrm{X}$ turno de trabalho de 8 horas X 22 dias X 12 meses X $80 \%$ da capacidade 
de produção (BRASIL, 2015A).

Para a análise da cobertura de mamografias, foi calculada a razão entre o número anual de exames realizados e o número estimado de mamografias necessárias na população de 40 a 69 anos, ajustada segundo a cobertura dos planos de saúde (Nm), e expressa em percentual. Um segundo indicador foi calculado considerando-se a diferença entre o número de mamógrafos disponíveis ao SUS e o número de mamógrafos estimados. Além disso, foi calculado o grau de utilização dos mamógrafos segundo sua capacidade de produção, do seguinte modo:

$$
\mathrm{Gu}=((\mathrm{Em} / \mathrm{Num}) / 5.069) \times 100
$$

\section{Em que:}

$\mathrm{Gu}$ = grau de utilização do mamógrafo (\%), dado pela razão entre a produção real do equipamento e a capacidade de produção do equipamento.

$\mathrm{Em}$ = número de mamografias realizadas segundo local de realização do exame.

Num = número de mamógrafos disponíveis ao SUS. (ADAPTADO DE BRASIL, 2015A, 2015B).

Após os cálculos, os dados foram organizados em tabelas segundo Regiões de Saúde, UF e Grandes Regiões. Os softwares utilizados para as tabulações foram o SPSS 22 e o ArcGIS 10.4 para a elaboração dos mapas.

\section{Resultados}

A tabela 1 apresenta, por Grandes Regiões e UFs, o número estimado (de acordo com as necessidades da população de mulheres de 40 a 69 anos) e o existente de mamógrafos disponíveis ao SUS segundo as 438 Regiões de Saúde definidas pelas secretarias estaduais de saúde. Observa-se que o parâmetro utilizado para o cálculo de mamógrafos sugere insuficiência em número de equipamentos nas Regiões de Saúde que compõem as regiões Norte, Nordeste e Centro-Oeste, enquanto as Regiões de Saúde do Sudeste e Sul apresentaram número de equipamentos maior do que o necessário segundo o parâmetro.

Embora o valor médio de mamógrafos estimado por Região de Saúde apresente relativa concordância com o número médio de equipamentos disponíveis ao SUS, observa-se concentração de equipamentos, quando se compara o número máximo de equipamentos estimados e disponíveis segundo Região de Saúde, como, por exemplo, no estado do Rio de Janeiro.

Tabela 1. Número e distribuição por Região de Saúde de mamógrafos estimados e mamógrafos disponíveis ao SUS segundo Grandes Regiões e Unidades da Federação, 2012

\begin{tabular}{|c|c|c|c|c|c|c|c|c|c|}
\hline \multirow{2}{*}{$\begin{array}{l}\text { Unidade da } \\
\text { Federação }\end{array}$} & \multirow{2}{*}{$\begin{array}{l}\begin{array}{l}\text { Regiões de } \\
\text { Saúde }\end{array} \\
\mathrm{N}\end{array}$} & \multirow{2}{*}{$\begin{array}{r}\begin{array}{l}\text { Mamógrafos } \\
\text { estimados }\end{array} \\
\mathrm{N}\end{array}$} & \multicolumn{3}{|c|}{$\begin{array}{c}\text { Distribuição por Região de } \\
\text { Saúde }\end{array}$} & \multirow{2}{*}{$\begin{array}{c}\text { Mamógrafos } \\
\text { disponíveis SUS } \\
\mathrm{N}\end{array}$} & \multicolumn{3}{|c|}{$\begin{array}{c}\text { Distribuição por Região de } \\
\text { Saúde }\end{array}$} \\
\hline & & & Média & Max & Min & & Média & Max & Min \\
\hline Brasil & 438 & 1.752 & 4 & - & - & 1.949 & 4 & - & - \\
\hline Norte & 45 & 117 & 3 & 17 & 0 & 109 & 4 & 29 & 1 \\
\hline Rondônia & 7 & 12 & 2 & 3 & 0 & 13 & 3 & 7 & 1 \\
\hline Acre & 3 & 5 & 2 & 3 & 0 & 3 & 3 & 3 & 3 \\
\hline Amazonas & 9 & 23 & 3 & 15 & 1 & 32 & 8 & 29 & 1 \\
\hline Roraima & 2 & 3 & 2 & 3 & 1 & 2 & 2 & 2 & 2 \\
\hline
\end{tabular}


Tabela 1. (cont.)

\begin{tabular}{|c|c|c|c|c|c|c|c|c|c|}
\hline Pará & 13 & 57 & 4 & 17 & 2 & 39 & 4 & 15 & 1 \\
\hline Amapá & 3 & 4 & 1 & 3 & 0 & 2 & 2 & 2 & 2 \\
\hline Tocantins & 8 & 15 & 1 & 2 & 1 & 18 & 3 & 10 & 1 \\
\hline Nordeste & 133 & 515 & 4 & 37 & 0 & 469 & 4 & 53 & 1 \\
\hline Maranhão & 19 & 58 & 3 & 11 & 2 & 48 & 3 & 11 & 1 \\
\hline Piauí & 11 & 32 & 3 & 11 & 0 & 32 & 4 & 12 & 1 \\
\hline Ceará & 22 & 82 & 4 & 21 & 1 & 51 & 3 & 11 & 1 \\
\hline $\begin{array}{l}\text { Rio Grande do } \\
\text { Norte }\end{array}$ & 8 & 30 & 4 & 9 & 1 & 31 & 4 & 18 & 1 \\
\hline Paraíba & 16 & 39 & 2 & 11 & 1 & 27 & 3 & 9 & 1 \\
\hline Pernambuco & 12 & 88 & 7 & 37 & 1 & 75 & 6 & 41 & 1 \\
\hline Alagoas & 10 & 28 & 3 & 10 & 1 & 38 & 5 & 25 & 1 \\
\hline Sergipe & 7 & 19 & 3 & 6 & 1 & 25 & 4 & 18 & 1 \\
\hline Bahia & 28 & 139 & 5 & 29 & 2 & 142 & 5 & 53 & 1 \\
\hline Sudeste & 153 & 701 & 5 & 82 & 1 & 842 & 6 & 81 & 1 \\
\hline Minas Gerais & 77 & 191 & 2 & 23 & 1 & 247 & 3 & 33 & 1 \\
\hline Espírito Santo & 4 & 30 & 8 & 14 & 4 & 30 & 8 & 16 & 4 \\
\hline Rio de Janeiro & 9 & 147 & 16 & 82 & 2 & 143 & 16 & 63 & 4 \\
\hline São Paulo & 63 & 333 & 5 & 72 & 1 & 422 & 7 & 81 & 1 \\
\hline Sul & 68 & 292 & 4 & 25 & 1 & 378 & 6 & 31 & 1 \\
\hline Paraná & 22 & 103 & 5 & 25 & 2 & 119 & 6 & 29 & 1 \\
\hline Santa Catarina & 16 & 65 & 4 & 9 & 2 & 91 & 6 & 13 & 1 \\
\hline Rio Grande do Sul & 30 & 124 & 4 & 22 & 1 & 168 & 6 & 31 & 1 \\
\hline Centro-Oeste & 39 & 127 & 3 & 19 & 0 & 151 & 5 & 32 & 1 \\
\hline $\begin{array}{l}\text { Mato Grosso } \\
\text { do Sul }\end{array}$ & 4 & 23 & 6 & 12 & 1 & 26 & 7 & 13 & 1 \\
\hline Mato Grosso & 16 & 26 & 2 & 7 & 0 & 28 & 3 & 13 & 1 \\
\hline Goiás & 18 & 60 & 3 & 16 & 0 & 78 & 5 & 32 & 1 \\
\hline Distrito Federal & 1 & 19 & 19 & 19 & 19 & 19 & 19 & 19 & 19 \\
\hline
\end{tabular}

Fonte: CNES/IBGE. 
A tabela 2 apresenta a cobertura de mamografias realizadas segundo necessidades na população de mulheres de 40 a 69 anos residentes, a diferença entre o número de mamógrafos disponíveis ao SUS e o número de mamógrafos estimados segundo o parâmetro do Ministério da Saúde e o grau de utilização do equipamento segundo UF e Grande Região.

Inicialmente, é importante destacar que, enquanto a cobertura de mamografias segundo necessidades é calculada em função da população residente, o grau de utilização do equipamento é calculado em função do local de realização do procedimento, incluindo parcelas de população residentes em outras regiões.

No Brasil, a cobertura de mamografia segundo a recomendação do Ministério da Saúde (BRASIL, 2015B) foi de 43,7\%. Somente cinco estados (Minas Gerais, Espírito Santo, São Paulo, Santa Catarina e Paraná) realizaram exames de mamografias acima de $50 \%$ da necessidade de suas populações. A cobertura de exames foi ainda menor na região Norte, onde apenas $18 \%$ da necessidade de exames da sua população foi atendida.

Quanto ao número de mamógrafos, observa-se que, no Brasil, no ano de 2012, existia um excedente de 196 mamógrafos. Os estados do Ceará, do Pará e de Pernambuco apresentaram deficit importante na quantidade de aparelhos, enquanto UFs das regiões Sudeste e Sul apresentaram número expressivo de equipamentos excedentes. As regiões Sul e Sudeste apresentaram o maior excedente de equipamentos no País. No Sudeste, observa-se que São Paulo apresenta o maior número de mamógrafos excedentes, com 89 unidades. No Sul, o estado do Rio Grande do Sul apresentou 44 equipamentos excedentes.

Com relação ao grau de utilização dos equipamentos, destacam-se os estados de São Paulo e do Paraná que apresentam, em média, utilização de cerca de $60 \%$ da capacidade dos aparelhos. As regiões Norte e Centro-Oeste apresentam grau de utilização em torno de $20 \%$. Para o total do País, o grau de utilização de um aparelho de mamografia foi de $44 \%$. A situação é mais complexa no Norte e Nordeste, com destaque especialmente na primeira região, com as 4 UFs com menor grau de utilização dos mamógrafos: Tocantins, Amapá, Pará e Rondônia. Os estados com maior grau de utilização dos equipamentos foram São Paulo, Paraná, Espírito Santo e Pernambuco. Destaque negativo neste aspecto é novamente o Rio de Janeiro, além do Rio Grande do Sul.

Tabela 2. Unidades da Federação segundo cobertura de mamografias na população de 40 a 69 anos residente, diferença entre o número de mamógrafos disponíveis ao SUS e o número de mamógrafos estimados, e grau de utilização dos mamógrafos SUS, 2012

\begin{tabular}{lrrr}
\hline Unidade da Federação & $\begin{array}{c}\text { \% cobertura de } \\
\text { mamografias segundo } \\
\text { necessidades 40-69 }\end{array}$ & $\begin{array}{c}\text { Diferença entre o número de } \\
\text { mamógrafos SUS e o número de } \\
\text { equipamentos estimados * }\end{array}$ & $\begin{array}{c}\text { \% grau de utilização dos } \\
\text { mamógrafos SUS }\end{array}$ \\
\hline Brasil & $\mathbf{4 3 , 7}$ & $\mathbf{1 9 6}$ & $\mathbf{4 4 , 2}$ \\
\hline Norte & $\mathbf{1 8 , 4}$ & $\mathbf{- 8}$ & $\mathbf{2 1 , 8}$ \\
\hline Rondônia & 18,2 & -2 & 18,0 \\
Acre & 21,7 & 9 & 40,0 \\
Amazonas & 38,0 & -1 & 30,9 \\
Roraima & 31,8 & & 53,0 \\
\hline
\end{tabular}




\begin{tabular}{|c|c|c|c|}
\hline Pará & 11,2 & -18 & 18,1 \\
\hline Amapá & 6,6 & -2 & 14,0 \\
\hline Tocantins & 14,2 & 6 & 10,5 \\
\hline Nordeste & 33,5 & -46 & 41,1 \\
\hline Maranhão & 18,4 & -10 & 25,9 \\
\hline Piauí & 22,6 & 0 & 25,0 \\
\hline Ceará & 23,5 & -31 & 41,6 \\
\hline Rio Grande do Norte & 28,4 & 1 & 31,1 \\
\hline Paraíba & 24,1 & -12 & 38,0 \\
\hline Pernambuco & 43,1 & -13 & 57,0 \\
\hline Alagoas & 42,9 & 10 & 37,2 \\
\hline Sergipe & 29,0 & 6 & 24,3 \\
\hline Bahia & 44,5 & 3 & 48,0 \\
\hline Sudeste & 54,6 & 141 & 51,3 \\
\hline Minas Gerais & 53,7 & 56 & 46,5 \\
\hline Espírito Santo & 51,4 & 0 & 58,2 \\
\hline Rio de Janeiro & 29,4 & -4 & 34,0 \\
\hline São Paulo & 66,4 & 89 & 59,5 \\
\hline Sul & 54,3 & 86 & 47,5 \\
\hline Paraná & 59,3 & 16 & 59,0 \\
\hline Santa Catarina & 62,3 & 26 & 49,2 \\
\hline Rio Grande do Sul & 45,9 & 44 & 28,4 \\
\hline Centro-Oeste & 24,2 & 24 & 22,2 \\
\hline Mato Grosso do Sul & 32,8 & 3 & 30,4 \\
\hline Mato Grosso & 20,2 & 2 & 19,9 \\
\hline Goiás & 25,1 & 18 & 21,1 \\
\hline Distrito Federal & 16,3 & 0 & 18,5 \\
\hline
\end{tabular}

Fonte: SIA/CNES.

* Número de equipamentos: valores inteiros. 
Para a análise das Regiões de Saúde, utilizaram-se como referência os valores nacionais da tabela 2. Assim, elas foram categorizadas segundo cobertura de mamografias realizadas em função da necessidade da população de mulheres de 40 a 69 anos residentes (abaixo e acima de $43,7 \%$ ); grau de utilização dos mamógrafos SUS segundo local de realização do exame (abaixo e acima de $44,2 \%$ ). Quanto à adequação do número de equipamentos de mamografias, as Regiões de Saúde foram classificadas em insuficientes ou excedentes, por meio do cálculo da diferença entre o número de equipamentos existentes disponíveis ao SUS e o número de equipamentos estimados.

No Brasil, 58,9\% das Regiões de Saúde apresentaram cobertura de exames abaixo da média nacional. Destacam-se as regiões Norte e Centro-Oeste, onde 97,8\% e 94,9\%, das Regiões de Saúde, respectivamente, não atingiram a média do País que é de $43,7 \%$ das residentes. No total, em 11 UFs, nenhuma Região de Saúde apresentou cobertura de mamografias em função da necessidade com valores percentuais acima da média no País. Na região Norte essa situação ocorre em 6 UFs.

Com relação ao número de equipamentos de mamografia, 47,5\% das Regiões de Saúde do Brasil apresentaram número de mamógrafos insuficientes. Nos estados do Acre, do
Amapá e de Roraima nenhuma das Regiões de Saúde apresentou o número esperado de equipamentos, enquanto no Nordeste, 90\% das Regiões de Saúde apresentaram número de equipamentos abaixo do sugerido pelo parâmetro. $\mathrm{O}$ estado que apresentou maior número de Regiões de Saúde com situação adequada quanto ao número de equipamentos foi Santa Catarina.

O grau de utilização por equipamento de mamografia ficou abaixo da média nacional em $67,4 \%$ das Regiões de Saúde. Entre estas, existem situações muito diversas, ocorrendo grau de utilização dos equipamentos acima da média no Sul e Sudeste. No Nordeste, algumas capitais e regiões do Norte baiano e do Oeste pernambucano apresentam grau de utilização mais elevado que a média nacional. No Norte, apenas uma região registrou grau de utilização acima da média nacional: Entorno de Manaus - o que pode ser um reflexo da concentração de equipamentos e do deslocamento de pessoas de outras regiões. Os estados de Pernambuco e de Santa Catarina apresentaram mais de dois terços de suas Regiões de Saúde com grau de utilização acima da média nacional. Além do Distrito Federal, outras seis UFs não apresentaram nenhuma Região de Saúde com grau de utilização acima da média do País (tabela 3).

Tabela 3. Número de Regiões de Saúde segundo cobertura de mamografias em mulheres de 40-69, diferença entre o número de mamógrafos SUS e o número de equipamentos estimados, grau de utilização dos mamógrafos SUS

\begin{tabular}{|c|c|c|c|c|c|c|c|c|c|c|c|c|}
\hline & \multicolumn{4}{|c|}{$\begin{array}{l}\text { Regiões segundo cobertura de } \\
\text { mamografias }\end{array}$} & \multicolumn{4}{|c|}{$\begin{array}{l}\text { Diferença entre o número de } \\
\text { mamógrafos SUS existentes e estimados }\end{array}$} & \multicolumn{4}{|c|}{$\begin{array}{c}\text { Regiões segundo grau de utilização dos } \\
\text { mamógrafos SUS }\end{array}$} \\
\hline & \multicolumn{2}{|c|}{$\begin{array}{l}\text { RS abaixo da } \\
\text { média nacional }\end{array}$} & \multicolumn{2}{|c|}{$\begin{array}{l}\text { RS acima da } \\
\text { média nacional }\end{array}$} & \multicolumn{2}{|c|}{$\begin{array}{l}\text { RS com falta de } \\
\text { equipamentos }\end{array}$} & \multicolumn{2}{|c|}{$\begin{array}{l}\text { RS com excedente } \\
\text { de equipamentos }\end{array}$} & \multicolumn{2}{|c|}{$\begin{array}{l}\text { RS abaixo da } \\
\text { média nacional }\end{array}$} & \multicolumn{2}{|c|}{$\begin{array}{l}\text { RS acima da } \\
\text { média nacional }\end{array}$} \\
\hline & $\mathbf{N}$ & $\%$ & $N$ & $\%$ & $\mathbf{N}$ & $\%$ & $N$ & $\%$ & $N$ & $\%$ & $\mathbf{N}$ & $\%$ \\
\hline Brasil & 258 & 58,9 & 180 & 41,1 & 208 & 47,5 & 230 & 52,5 & 295 & 67,4 & 143 & 32,6 \\
\hline Norte & 44 & 97,8 & 1 & 2,2 & 34 & 75,6 & 11 & 24,4 & 43 & 95,6 & 2 & 4,4 \\
\hline Rondônia & 7 & 100,0 & 0 & 0,0 & 4 & 57,1 & 3 & 42,9 & 7 & 100,0 & 0 & 0,0 \\
\hline Acre & 3 & 100,0 & 0 & 0,0 & 3 & 100,0 & 0 & 0,0 & 3 & 100,0 & 0 & 0,0 \\
\hline Amazonas & 8 & 88,9 & 1 & 11,1 & 7 & 77,8 & 2 & 22,2 & 9 & 100,0 & 0 & 0,0 \\
\hline
\end{tabular}


Tabela 3. (cont.)

\begin{tabular}{|c|c|c|c|c|c|c|c|c|c|c|c|c|}
\hline Roraima & 2 & 100,0 & 0 & 0,0 & 2 & 100,0 & 0 & 0,0 & 1 & 50,0 & 1 & 50,0 \\
\hline Tocantins & 8 & 100,0 & 0 & 0,0 & 4 & 50,0 & 4 & 50,0 & 8 & 100,0 & 0 & 0,0 \\
\hline Maranhão & 16 & 84,2 & 3 & 15,8 & 13 & 68,4 & 6 & 31,6 & 16 & 84,2 & 3 & 15,8 \\
\hline Piauí & 11 & 100,0 & 0 & 0,0 & 7 & 63,6 & 4 & 36,4 & 10 & 90,9 & 1 & 9,1 \\
\hline Ceará & 22 & 100,0 & 0 & 0,0 & 20 & 90,9 & 2 & 9,1 & 13 & 59,1 & 9 & 40,9 \\
\hline $\begin{array}{l}\text { Rio Grande do } \\
\text { Norte }\end{array}$ & 8 & 100,0 & 0 & 0,0 & 6 & 75,0 & 2 & 25,0 & 6 & 75,0 & 2 & 25,0 \\
\hline Pernambuco & 9 & 75,0 & 3 & 25,0 & 9 & 75,0 & 3 & 25,0 & 4 & 33,3 & 8 & 66,7 \\
\hline Alagoas & 9 & 90,0 & 1 & 10,0 & 6 & 60,0 & 4 & 40,0 & 8 & 80,0 & 2 & 20,0 \\
\hline Sergipe & 7 & 100,0 & 0 & 0,0 & 5 & 71,4 & 2 & 28,6 & 6 & 85,7 & 1 & 14,3 \\
\hline Bahia & 16 & 57,1 & 12 & 42,9 & 19 & 67,9 & 9 & 32,1 & 19 & 67,9 & 9 & 32,1 \\
\hline Sudeste & 43 & 28,1 & 110 & 71,9 & 46 & 30,1 & 107 & 69,9 & 86 & 56,2 & 67 & 43,8 \\
\hline Minas Gerais & 30 & 39,9 & 47 & 61,0 & 29 & 37,7 & 48 & 62,3 & 45 & 58,4 & 32 & 41,6 \\
\hline Espírito Santo & 1 & 25,0 & 3 & 75,0 & 1 & 25,0 & 3 & 75,0 & 2 & 50,0 & 2 & 50,0 \\
\hline Santa Catarina & 2 & 12,5 & 14 & 87,5 & 2 & 12,5 & 14 & 87,5 & 6 & 37,5 & 10 & 62,5 \\
\hline $\begin{array}{l}\text { Rio Grande do } \\
\text { Sul }\end{array}$ & 15 & 50,0 & 15 & 50,0 & 8 & 26,7 & 22 & 73,3 & 21 & 70,0 & 9 & 30,0 \\
\hline Centro-Oeste & 37 & 94,9 & 2 & 5,1 & 16 & 41,0 & 23 & 59,0 & 36 & 92,3 & 3 & 7,7 \\
\hline $\begin{array}{l}\text { Mato Grosso } \\
\text { do Sul }\end{array}$ & 4 & 100,0 & 0 & 0,0 & 1 & 25,0 & 3 & 75,0 & 4 & 100,0 & 0 & 0,0 \\
\hline Mato Grosso & 15 & 93,8 & 1 & 6,3 & 9 & 56,3 & 7 & 43,8 & 14 & 87,5 & 2 & 12,5 \\
\hline Goiás & 17 & 94,4 & 1 & 5,6 & 6 & 33,3 & 12 & 66,7 & 17 & 94,4 & 1 & 5,6 \\
\hline Distrito Federal & 1 & 100,0 & 0 & 0,0 & 0 & 0,0 & 1 & 100,0 & 1 & 100,0 & 0 & 0,0 \\
\hline
\end{tabular}

Fonte: SIA/CNES. 
Utilizando a categorização da tabela 3, foram construídos mapas que especializam o comportamento das variáveis cobertura de exames de mamografia, diferença entre o número de equipamentos existentes e o número de equipamentos estimados e grau de utilização dos mamógrafos segundo Regiões de Saúde (figura 1). Na figura 1-A, observa-se um percentual de mamografias superior à média nacional em áreas do Sul e Sudeste, assim como em algumas áreas do litoral e interior do Nordeste. Na figura 1-C, verifica-se que o grau de utilização dos mamógrafos é semelhante à cobertura observada na figura 1- $A$, destacando ainda mais a região Sudeste. Esse comportamento sugere que o percentual de realização de exames pode estar associado ao maior grau de utilização do equipamento. Por outro lado, a figura $1-B$ destaca que existem muitas Regiões de Saúde com excedente de mamógrafos, que, por sua vez, podem estar sendo subutilizados.

Figura 1. Regiões de Saúde segundo cobertura de mamografias em mulheres de 40-69 anos (Figura A), diferença entre o número de mamógrafos SUS e o número de equipamentos estimados (Figura B), e grau de utilização dos mamógrafos SUS (Figura C)

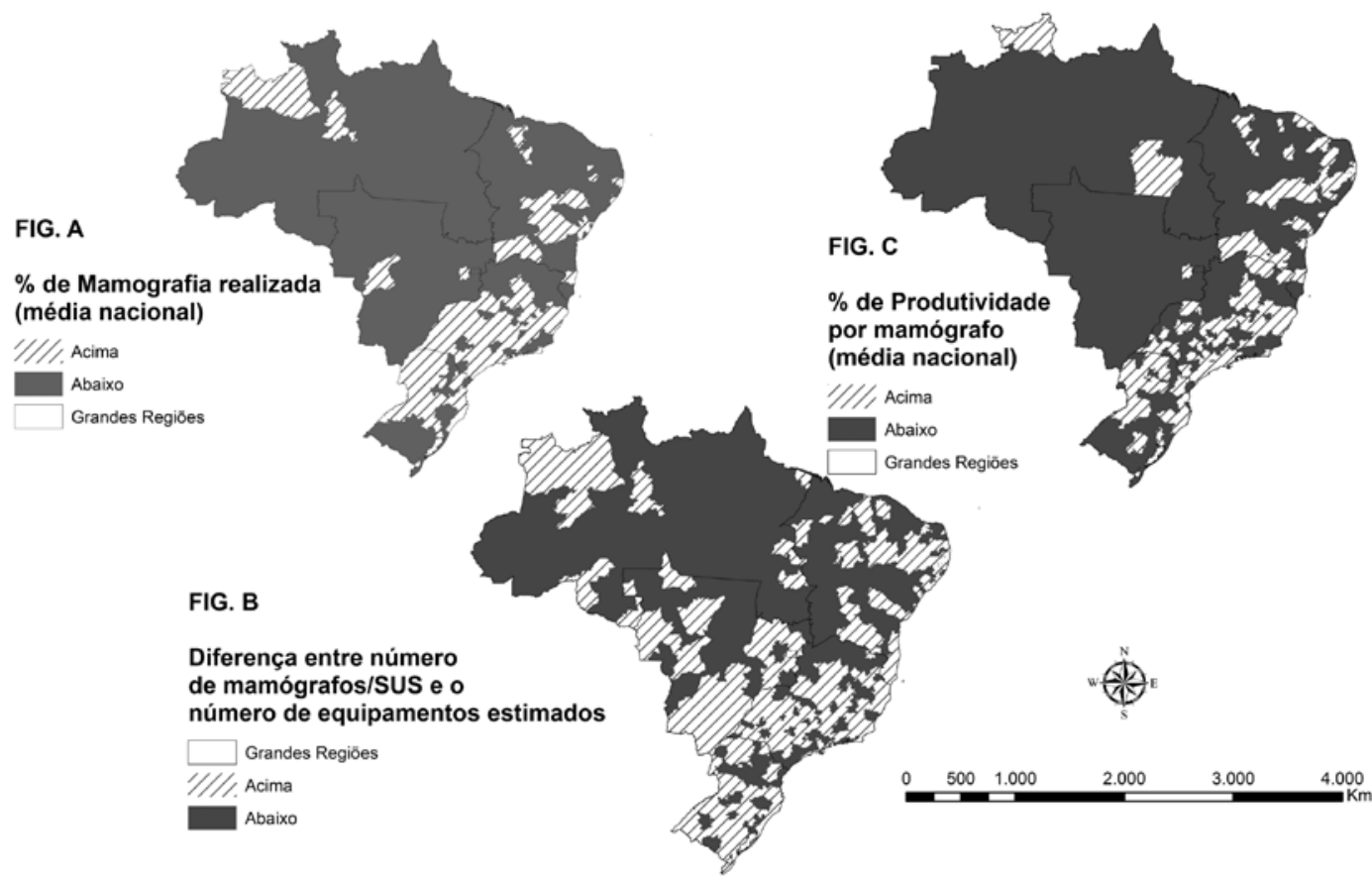

Fonte: Elaboração própria.

A figura 2 apresenta o comportamento das Regiões de Saúde segundo a cobertura de mamografias realizadas em função da necessidade de saúde da população em relação ao grau de utilização dos mamógrafos SUS.
Observa-se que, embora exista um coeficiente de determinação baixo e influenciado por valores aberrantes, a dispersão dos dados sugere que a realização de exames de mamografias na população pode estar 
associada a maior capacidade de utilização do equipamento. Ainda que a existência de muitos equipamentos ociosos já tenha sido destacada, os resultados apontam que em locais onde ocorre o maior aproveitamento da capacidade do equipamento também é maior a cobertura de exames nas populações. Contudo, a interpretação da figura requer cautela, já que para o percentual de mamografias realizadas foram considerados exames na população residente e no percentual de utilização do equipamento é considerado o local de realização do exame.

Figura 2. Gráfico de dispersão da cobertura de mamografias em mulheres de 40-69 anos em relação ao grau de utilização dos mamógrafos SUS

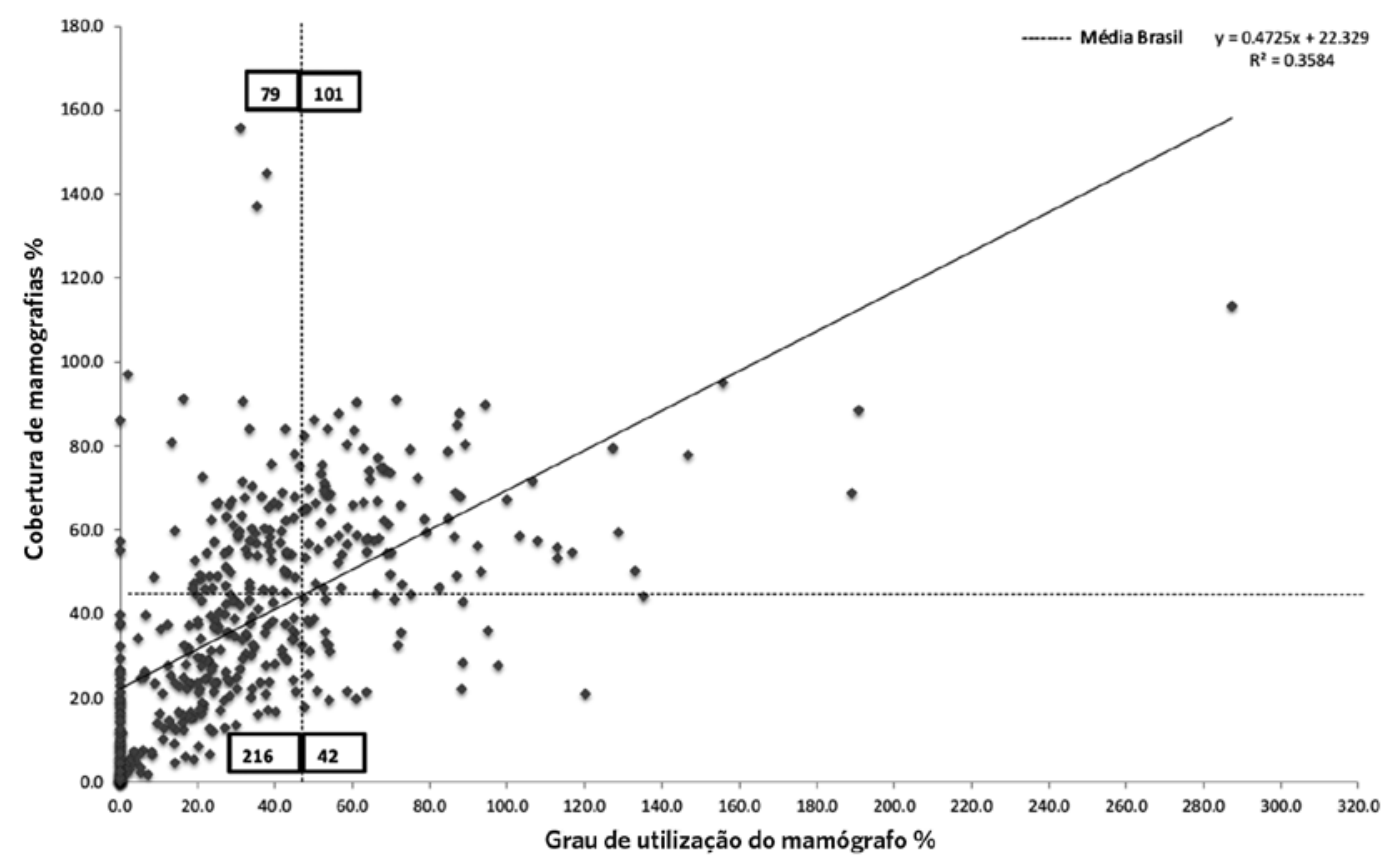

Fonte: Elaboração própria.

No gráfico da figura 2, observa-se a concentração das Regiões de Saúde na interseção dos eixos. Isso indica situações de baixa cobertura entre a população-alvo aliada ao baixo grau de utilização dos mamógrafos disponíveis. Um total de 216 regiões, equivalente a 49,3\%, se enquadra nessa situação, destacando-se especialmente as regiões Norte, Nordeste e Centro-Oeste, apesar de ser presente em todas as grandes regiões do País. A segunda classe que mais se destaca é marcada por situações com cobertura e grau de utilização dos equipamentos acima da média nacional, com 101 (23,1\%) Regiões de Saúde, estas concentradas nas regiões Sudeste e Sul. Situações de alta cobertura com baixo grau de utilização ocorrem em 79 (18\%) Regiões de Saúde e, neste caso, também prevalecem nas regiões do Sul e Sudeste. O grupo com menor concentração de Regiões de Saúde é o de baixa cobertura com grau de utilização acima da média, com apenas $42(9,6 \%)$ do total de regiões, sendo que 20 se localizam no Nordeste. 


\section{Discussão}

O rastreamento para detecção precoce do câncer de mama é fundamental para garantir o tratamento oportuno às mulheres vítimas desse tipo de neoplasia, que é a que mais afeta essa população no Brasil, inclusive de maneira crescente no período recente (SILVA, 2012). A análise dos indicadores propostos neste estudo demonstrou a diversidade de situações de disponibilidade/utilização dos equipamentos de mamografia referentes à organização do serviço que podem implicar dificuldades para estabelecer uma política efetiva de detecção precoce para a população usuária do SUS, questão já apontada por autores como Oliveira et al. (2011) e Silva et al. (2014), tais como a falta de equipamentos disponíveis e de profissionais habilitados, subutilização de equipamentos e dificuldade de acesso em função das distâncias geográficas.

De modogeral, pode-se apontar que há uma situação de baixa cobertura para a população-alvo no âmbito do SUS, já que apenas 43,7\% dos exames esperados segundo a necessidade foram realizados. Dados da Organização para Cooperação e Desenvolvimento Econômico (OECD) para o ano de 2011 apontam cobertura média de exames de mamografia para $61,5 \%$ das mulheres entre 50 e 69 anos em um grupo de 33 países (OECD, 2013). As comparações da cobertura de exames de mamografia verificada no presente estudo colocam o Brasil à frente de países como México, Chile, Japão e Turquia. Entretanto, cabe interpretar com cautela os dados aqui apresentados, já que aqueles dos países da OECD são coletados segundo diferentes metodologias para mulheres de 50 a 69 anos. No Brasil, dados da Pesquisa Nacional de Saúde (PNS, 2013) obtidos por meio de inquérito populacional apontam cobertura de $60 \%$ da população feminina de 50 a 69 anos.

Os resultados deste trabalho sugerem relação entre a baixa cobertura da população e aspectos ligados a condições socioeconômicas das Regiões e estados. São expressões desse fato as piores situações da região Norte em geral, dos estados do Maranhão e do Piauí no contexto do Nordeste e das Regiões de Saúde do Vale do Jequitinhonha e norte de Minas Gerais no âmbito do Sudeste. LimaCosta e Matos (2007) apontam os problemas de acesso à mamografia no nível individual em função da situação socioeconômica, o que também foi ressaltado por Oliveira et al. (2011). Além disso, há que se considerar a dificuldade de acesso ao exame em função da falta de indicação por um médico, questão também apontada por estes últimos autores, tanto no caso da necessária realização em determinados grupos etários como da própria continuidade do rastreamento ao longo do tempo.

O número de mamógrafos no País apresenta um excedente de 196 equipamentos em relação ao que seria necessário de acordo com os parâmetros, porém com uma distribuição bastante heterogênea e, como agravante, baixo grau de utilização. A questão da cobertura de mamografia é diretamente afetada pela disponibilidade e pela distribuição dos mamógrafos. Citando documento da Sociedade Brasileira de Mastologia (SBM - www.sbmastologia.com.br), Oliveira et al. (2011) apontam a distribuição inadequada de equipamentos como um dos principais desafios à cobertura da população. Se para o total nacional há mais mamógrafos do que seria necessário, novamente a situação de estados e Regiões de Saúde é bastante diversa. No nível das grandes regiões, o Sudeste e o Sul se destacam pelo volume de equipamentos acima do que seria adequado de acordo com os parâmetros. Com relação aos estados, a pior situação se encontra no Norte e Nordeste, com destaque para o deficit de equipamentos no Ceará, em Pernambuco e no Pará. Novamente, o Rio de Janeiro registra situação problemática, sendo a única UF com falta de equipamentos no Centro-Sul do País.

Quando se observam as Regiões de Saúde, verifica-se que a maioria delas está adequada, ou seja, não é a falta de equipamentos que explica a cobertura abaixo do recomendado. 
As situações mais problemáticas se concentram no Pará, no oeste do Maranhão, Norte de Minas Gerais e região central da Bahia. No Sudeste e Sul, há regiões isoladas com deficit de equipamentos, especialmente nas regiões interioranas. Vazios ocorrem marcadamente no Norte, além da região Norte de Mato Grosso. São Regiões de Saúde em que não há equipamentos, e as mulheres usuárias do SUS têm que se dirigir a outras regiões para realizar o exame.

Em algumas regiões, pode-se apontar a falta de equipamentos como causa para a baixa cobertura de mamografias, especialmente no Norte e em algumas regiões interioranas das outras partes do País. A problemática distribuição dos equipamentos foi apontada por autores como Lima-Costa e Matos (2007), Oliveira et al. (2011) e Silva et al. (2014) como geradora da necessidade de grandes deslocamentos para a realização de mamografias, marcante especialmente na região Norte e mesmo em direção aos grandes centros urbanos nas outras regiões do País. Todavia, há que se apontar como parte dos desafios a própria disponibilidade de recursos humanos e físicos para instalação, manutenção e operação dos mamógrafos, que ampliam a questão da sua distribuição.

O baixo grau de utilização dos mamógrafos em relação à sua capacidade de produção expressa dificuldades para além da distribuição geográfica dos equipamentos. A média nacional do grau de utilização dos mamógrafos está abaixo da metade da capacidade dos equipamentos. Esse comportamento relacionado com a cobertura da população, utilização e produção do mamógrafo pode ocorrer de forma sistemática em outros procedimentos diagnósticos. Santos et al. (2014) avaliaram o grau de utilização dos equipamentos de tomografia computadorizada no Brasil e observaram que ele é baixo.

Ao associar o grau de utilização à cobertura de mamografias, é possível destacar alguns pontos no comportamento dos quatro grupos classificados segundo as médias nacionais.
Nas regiões onde a cobertura e o grau de utilização são altos, ocorre uma relação direta entre estes dois indicadores, assim como nas regiões onde ambas são baixas.

Nas regiões onde a cobertura é alta e o grau de utilização é baixo, destacam-se alguns fatores que podem elucidar esse comportamento. Em 77 Regiões de Saúde, o grau de utilização é zero, destas, 54 não tinham mamógrafos SUS. Possivelmente, o comportamento das 23 regiões que dispõem de equipamentos e não apresentaram produção pode estar relacionado a problemas com as informações do CNES quanto ao uso ou à ausência de profissionais habilitados para realização do exame, como destaca Gutierrez (2009). Em algumas dessas regiões sem a presença de equipamentos e onde a cobertura é elevada, como por exemplo, a Região de Seabra (BA), ocorre a utilização de mamógrafos móveis.

Em regiões onde ocorre alto grau de utilização e baixa cobertura, é possível que exista baixo número de equipamentos, o que pode sobrecarregar os existentes. Nesses casos, seria interessante verificar a viabilidade de aumentar o número de mamógrafos. Outra questão importante é que essas regiões podem estar recebendo pacientes não residentes. Contudo, deve-se observar esses resultados com parcimônia, já que foi verificado em algumas Regiões de Saúde número de equipamentos insuficientes para o número de exames realizados, como, por exemplo, as regiões de Garanhuns (PE) e Caicó (RN).

A situação da cobertura do rastreamento para detecção precoce do câncer de mama em mulheres de 40 a 69 anos é problemática, e não se pode apontar a falta de equipamentos como a causa única e direta dos problemas. Possivelmente, a distribuição dos equipamentos entre as unidades geográficas, associada à disponibilidade de recursos humanos para operação dos equipamentos e mesmo o acesso à recomendação de realização do exame, também se enquadra no 
conjunto de questões relacionadas com as lacunas do rastreamento (ELKIN ET AL., 2010).

Para além das desigualdades socioeconômicas e geográficas, há uma complexa articulação de características que ampliam o caráter desafiador da questão. Comparar as situações registradas pelo Rio Grande do Sul e por Pernambuco é bastante interessante nesse contexto. $\mathrm{O}$ primeiro tem excedente de mamógrafos e baixo grau de utilização, enquanto o último registra a situação contrária: falta de mamógrafos e grau de utilização acima da média nacional, entre as mais significativas do País. A cobertura da população de acordo com as necessidades se assemelha entre os dois estados.

Para tornar efetivas as ações de rastreamento para detecção precoce do câncer de mama, é necessário que sejam levadas em conta as variações acima registradas. Não basta investir em equipamentos, é necessário ampliar a disseminação de informações sobre a importância da mamografia, mas principalmente dos recursos humanos envolvidos na solicitação do exame, na sua interpretação, além da operação e manutenção dos equipamentos. Especialmente no âmbito das Regiões de Saúde, como unidade espacial de organização da atenção, é necessário considerar articulações que busquem superar desafios e, assim, ampliar a atenção a uma demanda estabelecida em parâmetros nacionais e internacionais, não como obrigação do cumprimento de metas, mas sim como um compromisso com os princípios constitucionais do SUS.

É importante destacar as limitações deste estudo, relacionadas especialmente com as fontes das informações utilizadas. A situação atual pode ter apresentado alterações em relação a 2012, ano considerado na análise. No entanto, até o momento da elaboração deste estudo, os dados de projeções populacionais por faixas etárias elaborados pelo IBGE só estavam disponíveis até esse ano. Apesar das fragilidades já identificadas em outros trabalhos que utilizaram dados do
CNES como Gutierrez et al. (2009) e Santos et al. (2014), este estudo optou pela sua utilização, dada a inexistência de outras fontes de dados para avaliação das Regiões de Saúde, contudo suas limitações são consideradas na abordagem das questões aqui desenvolvidas. Também é interessante destacar o fato de que os dados sobre cobertura se baseiam na população residente, enquanto o grau de utilização dos equipamentos diz respeito ao local de ocorrência. Como já indicado na seção de métodos, essa distinção foi considerada na análise, evitando que comparações ou interpretações fossem prejudicadas. Para contornar esse problema, seria ideal considerar a população potencialmente coberta pelo local de realização de exame (MAGALHÃES ET AL., 2015). Entretanto, esse é um indicador de difícil construção que, no caso da unidade de análise objeto do presente estudo, poderia prejudicar a acurácia dos resultados. Ainda como limitação, ressalte-se que não foi utilizado o parâmetro de distância mínima de acesso ao exame devido à unidade de análise utilizada no presente estudo.

Dada a diversidade de situações quanto à cobertura de mamografia e à disponibilidade e utilização dos equipamentos, não existe uma única solução enquanto política de saúde. O procedimento de mamografia é considerado um exame diagnóstico de média complexidade. A sua natureza possibilita estratégias de cobertura que vão desde campanhas nacionais e mutirões até o deslocamento de equipamentos e pessoal a locais mais remotos. Contudo, este estudo aponta que o gerenciamento racional e otimizado de recursos físicos e, certamente, de recursos humanos pode solucionar algumas questões imediatas. Em longo prazo, um caminho interessante é a articulação entre as diferentes esferas governamentais. Nessa perspectiva, a regionalização pode significar o uso mais eficiente de recursos, a ampliação do acesso e a melhor qualidade da atenção à saúde (KUSCHNIR; CHORNY, 2010). Assim, deve-se considerar que a regionalização não se baseie 
apenas na delimitação física do espaço geográfico em função da esfera administrativa, mas ponderar também questões como acesso geográfico e a rede de referência e contrarreferência de serviços (GONDIM ET AL., 2008).

A utilização de parâmetros específicos para o cálculo de equipamentos e recursos humanos é uma prerrogativa válida e necessária para o planejamento do atendimento de saúde. Entretanto, devem ser incorporados mecanismos de análise de produção e utilização dos serviços e equipamentos vinculados à solicitação e disponibilização dos recursos. Sem esses mecanismos de avaliação, recursos podem estar sendo subdimensionados ou superdimensionados e, consequentemente, subutilizados.

\section{Referências}

AGÊNCIA NACIONAL DE SAÚDE SUPLEMENTAR

(ANS). Informações em saúde suplementar. Disponível em: <http://www.ans.gov.br/anstabnet/cgi-bin/ dh?dados/tabnet_02.def >. Acesso em: 16 mar. 2016.

BRASIL. Ministério da Saúde. Decreto 7.508, de 28 de junho de 2011. Regulamenta a lei ${ }^{\circ}$. 8.080, de 19 de setembro de 1990, para dispor sobre a organização do SUS, o planejamento da saúde, a assistência à saúde e a articulação interfederativa, e dá outras providências. Diário Oficial [da] União, Brasília, DF, 2011. Disponível em: <http://www.planalto.gov.br/ccivil_03/_ato20112014/2011/decreto/D7508.htm>. Acesso em: 08 set. 2016.

Ministério da Saúde. Instituto Nacional de Câncer. Parâmetros para o rastreamento do câncer de mama: recomendações para gestores estaduais e municipais. Rio de Janeiro: INCA, 2009. 13 p.

Ministério da Saúde. Instituto Nacional de Câncer. Divisão de Detecção Precoce e Apoio à Organização de Rede Coordenação de Prevenção e Vigilância. Nota Técnica Revisão do Parâmetro para Cálculo da Capacidade de Produção do Mamógrafo Simples, 2015a. Disponível em: <http://www2.inca.gov.br/wps/wcm/connect/ fb4249004a99e827be5dfe504e7bf539/Nota+Revis\%C3 $\%$ A3o+Capacidade+dos+Mam\%C3\%B3grafos+2015.pdf ?MOD=AJPERES\&CACHEID=fb4249004a99e827be 5 dfe504e7bf539>. Acesso em: 24 jul. 2016.

Ministério da Saúde. Portaria GM/MS no 4.279, de 30 de dezembro de 2010. Estabelece diretrizes para a organização da Rede de Atenção à Saúde no âmbito do Sistema Único de Saúde (SUS). Diário Oficial [da] União, Brasília, DF: Ministério da Saúde, 31 dez. 2010. Disponível em: <http://bvsms.saude.gov.br/ bvs/saudelegis/gm/2010/prt4279_30_12_2010.html>. Acesso em: 08 set. 2016.

Ministério da Saúde. Secretaria de Atenção à Saúde. Departamento de Regulação, Avaliação e Controle de Sistemas. Critérios e Parâmetros para o Planejamento e Programação de Ações e Serviços de Saúde no âmbito do Sistema Único de Saúde. Brasília, DF: Ministério da Saúde, 2015b. Disponível em: < http://www.fehosp.com.br/app/ webroot/files/manuais/5ffa8dle03f7edb0leleed7b07178c fb.pdf>. Acesso em: 31 ago. 2016.

ELKIN, E. B. et al. Geographic access and the use of screening mammography. Medical care, Philadelphia, v. 
48, n. 4, p. 349-356, 2010.

GEBRIM, L. H.; QUADROS, L. G. A. Rastreamento mamográfico no Brasil. Revista Brasileira de Ginecologia e Obstetrícia, Rio de Janeiro, v. 28, p. 319323, 2006.

GONDIM, G. M. et al. O território da saúde: a organização do sistema de saúde e a territorialização. In: MIRANDA, A. C. et al. (Org.). Território, ambiente e saúde. Rio de Janeiro, Fiocruz, 2008. p.183-203.

GUTIERREZ M. A oferta de tomógrafo computadorizado para o tratamento do acidente vascular cerebral agudo, no Brasil, sob o ponto de vista das desigualdades sociais e geográficas. 2009. $61 \mathrm{f}$. Dissertação (Mestrado em Saúde Pública) - Escola Nacional de Saúde Pública Sergio Arouca, Fundação Oswaldo Cruz, Rio de Janeiro, 2009.

\section{INSTITUTO BRASILEIRO DE GEOGRAFIA E} ESTATÍSTICA (IBGE). Pesquisa Nacional de Saúde 2013: percepção do estado de saúde, estilos de vida e doenças crônicas: Brasil, Grandes Regiões e Unidades da Federação. Rio de Janeiro: Instituto Brasileiro de Geografia e Estatística. 2014. Disponível em: <ftp://ftp.ibge.gov.br/ PNS/2013/pns2013.pdf>. Acesso em 31 jan. 2015.

KUSCHNIR, R.; CHORNY, A. H. Redes de atenção à saúde: contextualizando o debate. Ciência e Saúde Coletiva, Rio de Janeiro, v. 15, n. 5, p. 2307-2316, 2010.

LIMA-COSTA, M. L.; MATOS, D. L. Prevalência e fatores associados à realização da mamografia na faixa etária de 50-69 anos: um estudo baseado na Pesquisa Nacional por Amostra de Domicílios (2003). Cad. Saúde Pública, Rio de Janeiro, v. 23, n. 7, p. 1665-1673, jul, 2007.

LIMA, L. D. et al. Regionalização e saúde no Brasil. In: GIOVANELLA, L. et al. (Org.). Políticas e Sistema de Saúde no Brasil. 2 ed. Rio de Janeiro: Editora Fiocruz, 2012. p. 823-852.

MAGALHÃES, M. A. F. M; XAVIER, D. R.; MATOS,

V. Projeto Saúde Amanhã. Relatório de Pesquisa Sobre Definição de área de abrangência e denominador populacional para polos de atendimento municipal segundo fluxo de pacientes. Agosto, 2015. Disponível em: <http:// saudeamanha.fiocruz.br/wp-content/uploads/2016/07/ rp-4MAGALH\%C3\%83ES-Monica-et-al-\%C3\%81REAABRANG\%C3\%8ANCIA-E-DENOMINADORPOPULACIONAL-POLOS-ATENDIMENTOMUNICIPAL.pdf $>$. Acesso em: 8 set. 2016.

OLIVEIRA, E. X. G. et al. Condicionantes socioeconômicos e geográficos do acesso à mamografia no Brasil, 2003-2008. Ciência \& Saúde Coletiva, Rio de Janeiro, v. 16, n. 9, p. 3649-3664, 2011.

\section{ORGANIZATION FOR ECONOMIC COOPERATION} AND DEVELOPMENT (OECD). Health at a glance 2013. Paris: OECD Publishing, 2013. Disponível em: $<$ https://www.oecd.org/els/health-systems/Health-ata-Glance-2013.pdf >. Acesso em: 31 ago. 2016.

SANTOS, D. L. D. et al. Capacidade de produção e grau de utilização de tomógrafo computadorizado no Sistema Único de Saúde. Cad. Saúde Pública, Rio de Janeiro, v. 30, n.6, p. 1293-1304, 2014.

SILVA, G. A. Câncer de mama no Brasil: estratégias para o seu enfrentamento. Cad. Saúde Pública, Rio de Janeiro, v. 28, n.1, p. 4-5, jan. 2012.

SILVA, G. A. et al. Acesso à detecção precoce do câncer de mama no Sistema Único de Saúde: uma análise a partir dos dados do Sistema de Informações em Saúde. Cad. Saúde Pública, Rio de Janeiro, v. 30, n. 7, p. 15371550, jul. 2014.

VIACAVA, F. et al. Avaliação de Desempenho de Sistemas de Saúde: um modelo de análise. Ciência e Saúde Coletiva, Rio de Janeiro, v. 17, p. 921-934, 2012.

VIACAVA, F. et al. Uma metodologia de avaliação do desempenho do sistema de saúde brasileiro. Ciência \&t Saúde Coletiva, Rio de Janeiro, v. 9, n. 3, p. 711-724, 2004.

Recebido para publicação em abril de 2016

Versão final em agosto de 2016

Conflito de interesses: inexistente

Suporte financeiro: não houve 